Jpn. J. Hyg. , 48, 578-585 (1993)

\title{
Statistical Analysis and Prediction on Incidence of Infectious Diseases Based on Trend and Seasonality
}

\author{
Masayuki KAKEHASHI, Satoko TSURU, Akihiko SEO, \\ Ahmed AMRAN and Fumitaka YOSHINAGA
}

\author{
Department of Public Health, Hiroshima University School of Medicine, Hiroshima
}

\begin{abstract}
We proposed a prediction methodology for the incidence of infectious diseases using incidence data on measles and influenza for forty years in Japan. We also proposed a diagram that makes it possible to convey information on infectious disease incidence more attractively to a wider audience. This can be a useful tool for health promotion in the community .

The obtained results are as follows:

1. It was advantageous to use data transformed by logarithm in statistical analysis of infectious disease incidence.

2. The incidences of measles and influenza exhibited strong seasonality. Measles was most frequent in June and influenza in February .

3 . Long-term trends were extracted from the derived data obtained by eliminating seasonal effects from the original data. For measles, a decline was accelerated by the introduction of vaccination program in 1978. Influenza also showed a decline for these thirty years.

4 . The observed incidence data were quite well predicted by only the trend and the seasonality . The squares of multiple regression coefficients of measles and influenza were 0.84 and 0.58 , respectively. The analysis of the residuals suggested there was a possibility of improvement in prediction.

5. The improvement in prediction was attained by incorporating an autoregressive component of the residuals. As a result, the squares of multiple correlation coefficients of measles and influenza increased to 0.97 and to 0.79 , respectively.

6. We finally proposed the TS-decomposition diagram to facilitate practical use of incidence data. In this diagram, current incidence data and predicted values for the near future are plotted on the plane where the trend and the seasonality are superimposed.

We also discussed the application of our method to the entire range of infectious disease surveillance data.
\end{abstract}

Key words : Incidence of infectious diseases, Prediction of incidence, Measles, Influenza, TS-decomposition diagram

\section{Introduction}

Infectious diseases have had a great effect on human history. Most of the earlier public health activities were devoted to prevention from these diseases. By development of medical technology, e.g., vaccination and antibiotics, and improvement in nutrition and environmental sanitation, the danger from infectious diseases has been lowered. Public health systems have been expanded and are sophisticated in carrying out such activities. Prevention of infectious diseases is, however, still very important to children and the elderly. Among the various kinds of preven-

Reprint requests to: Masayuki Kakehashi, PhD, Department of Public Health, Hiroshima University Scool of Medicine, Kasumi, Minami-ku, Hiroshima 734, Japan 
tive efforts, a surveillance system of infectious disease incidence is expected to play an important role in infectious disease control.

It is almost a decade since the nation-wide surveillance system of infectious diseases started in Japan. Though some information on the incidence of infectious diseases has been reported to general practitioners in Hiroshima, their level of interest was low according to our questionaire survey ${ }^{1,2)}$.

In this paper, we tried to establish a prediction methodology for the incidence of infectious diseases and presented a diagram that enables us to convey information on infectious disease incidence more attractively to a wider audience.

\section{Materials and Methods}

We used incidence data on measles and influenza for forty years (1950-1989) in Japan. The sources of data were Statistics on Communicable Diseases Japan for $1971^{3)}$ and $1989^{4)}$, in which

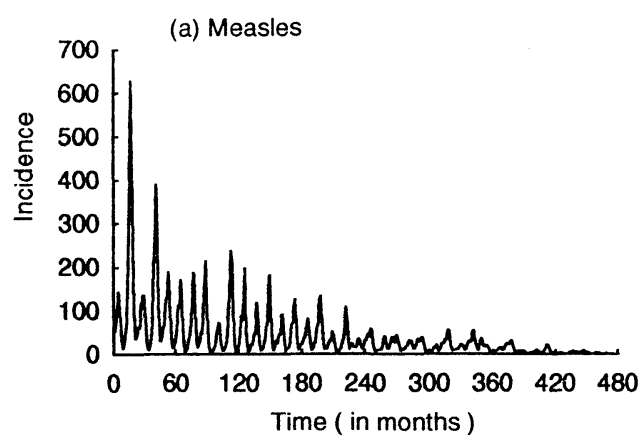

which the data were presented case rates per 100,000 population by month. We can eliminate the effect of changes in population size by using case rates rather than case numbers. Thus, we had two time series of 480 sampling points. The data are plotted in Fig. 1 and in Fig. 2. We chose months as the time unit $(1 \leqq \mathrm{t} \leqq 480)$ and utilized optical character recognition (OCR) sof tware for data input.

We also used incidence data on measles and influenza in $1990^{5)}$ to test external validity of our prediction models.

The analyses in this study were mainly carried out with a statistical analysis package, SYSTAT ${ }^{6)}$, on a personal computer.

We first performed tentative regression analyses of incidence by year and month to estimate benefit of logarithmic transformation of the data. The dependent variables were incidence and logarithm of incidence, while explanatory variables were effects of years (40 variables) and

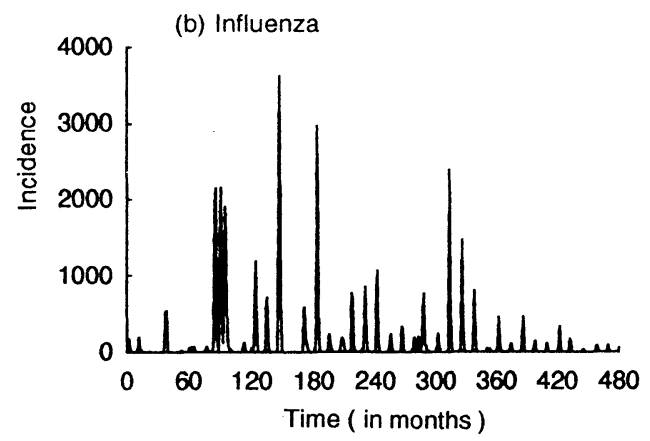

Fig. 1 Incidence (case rates per 100,000 population) during 1950-1989

(a) Measles

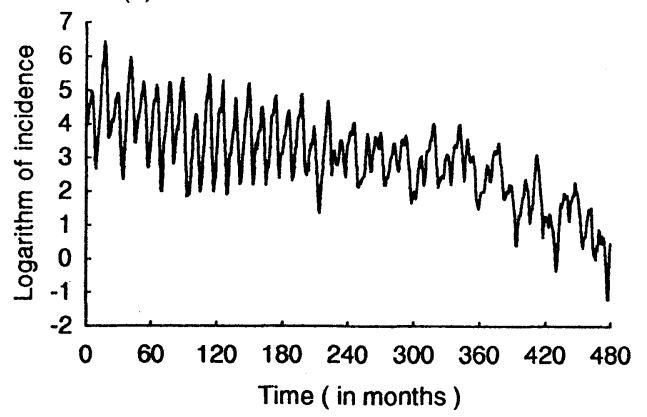

(b) Influenza

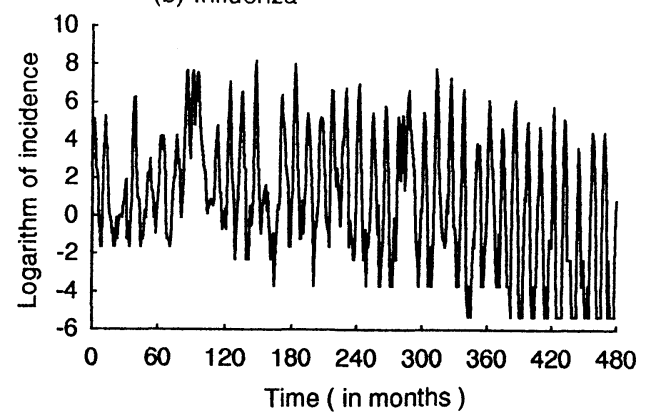

Fig. 2 Incidence during 1950-1989 in logarithmic scale 
months (12 variables) for each infectious disease. Note here that zero valued samples were neglected in the analysis for the logarithm of incidence of influenza.

When we transform a variable by logarithm, trouble will arise if the variable has a zero value. In our study, the influenza incidence data provided an example. Of the zero values, some were rounded zeros (magnitude less than half of the unit employed) and others were absolute zeros (magnitude zero). In our analyses below, we transformed the rounded zeros to $-3.689\left(=\log _{\mathrm{e}}\right.$ $0.025)$ and absolute zeros to $-5.298\left(=\log _{\mathrm{e}}\right.$ $0.005)$. These values were selected as ones that maximized the multiple correlation coefficient of tentative regression analysis out of several combinations of candidate values. The value for the absolute zero was different from that in the earlier version of this work ${ }^{7)}$ while the one for the rounded zero was the same.

We next analyzed seasonality and the longterm trend of the data. Investigation on seasonality was carried out by using the seasonal procedure in the series menu of SYSTAT, adopting an additive model. The long-term trend was extracted by the LOWESS procedure in the series menu in SYSTAT. LOWESS is a smoothing method using weighted least squares ${ }^{8)}$. The tension factor used in the analyses was 0.5 , which is the default value in SYSTAT. The tension factor specifies the range for calculating weighted sums and works like tension for the trend curves. Straighter trend curves result from large values of the tension factor, or from wide ranges, whereas twisted trend curves more faithful to the original data are obtained wih small values for the tension factor, or within narrow ranges. Long-term trends were calculated using the nonlin menu in SYSTAT according to the shapes suggested by the results of LOWESS.

Making use of the obtained results, we constructed primary prediction models and evaluated the goodness of fit. The residuals were analyzed by the autocorrelation method for time series analysis. We elaborated the primary prediction models according to the results of residual analysis by incorporating an autoregression component into the models. The degree of fitness of the prediction was also tested by using external data (data not used in model construction), i.e., incidences of measles and influenza in $1990^{5}$ ).

\section{Results}

\section{Tentative Regression Analyses}

We first performed regression analyses of incidence by year and month to estimate the benefits of logarithmic transformation of the data. The results are summarized in Table 1 . The multiple correlation coefficients were greatly increased by logarithmic transformation of the data. This is consistent with the common knowledge that logarithmic transformation is of ten useful in biomedical data analysis. Therefore, we decided to use logarithmic transformation.

Table 1 Coefficients of determination (squares of multiple correlation coefficients) obtained in the tentative regression analysis

\begin{tabular}{ccc}
\hline dependent variable & measles & influenza \\
\hline incidence & 0.628 & 0.372 \\
logarithm of incidence & 0.904 & 0.626 \\
\hline
\end{tabular}

\section{Seasonality}

The observed incidence data for both measles and influenza exhibited apparent seasonality . The obtained seasonality indices are shown in Fig. 3. Because we adopted an additive model, the effect of seasonality was added to the logarithm of incidence. This can be interpreted to be a multiplying factor after inverse transformation. Thus, if seasonality index is two, incidence is twice as large as the average. In Fig. 3 , the geometric mean is set as unity .

Measles had a peak in June, a bottom in October. Influenza was most frequent in February, whereas it was least frequent around August. By subtracting the seasonality index from the original data, we acquired season-adjust ed time series to examine long-term trends more precisely . 


\section{Long-Term Trends}

The results are shown in Fig.4. In measles, the trend is expressed by two segments that bend at near $t=350$. After this point, tendency of decline is accelerated. Because the acceleration of decline is attributable to the vaccination program introduced in October, 1978 (at $\mathrm{t}=346$ ), we concluded that the long-term trend for measles can be expressed by line segments that bend at $\mathrm{t}=346$. We calculated the long-term trend for measles by piecewise linear regression. The square of the correlation coefficient $\left(R^{2}\right)$, or coefficient of determination was 0.610.

For influenza, the long-term trend was different from that of measles. In this case, the trend seemed to be a hump having a peak at ca. $t=140$, i.e., about 1962 . Though we could not find any apparent reason why it humped like this,

(a) Measles

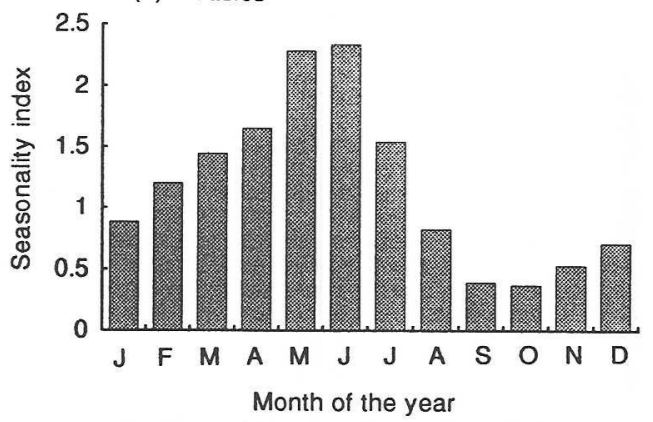

we determined to adopt a quadratic curve as the long-term trend for influenza incidence and we calculated this long-term trend by quadratic regression. The $\mathrm{R}^{2}$ was 0.149 .

\section{Primary Prediction Models}

To what extent can we predict the observed incidence pattern by using a model simply involving seasonality and the long-term trend? The square of the multiple correlation coefficient $\left(R^{2}\right)$, or coefficient of determination, was almost satisfactory, being 0.84 for measles and 0.58 for influenza. We call this primary prediction. The residuals were analyzed by an autocorrelation method for time series analysis. The effects of lags 1, 2, 13 and 25 for measles were suggested from the partial autocorrelation function plot shown in Fig. 5 (a). In the case of influenza, the effect of lag 1 was strongly suggested

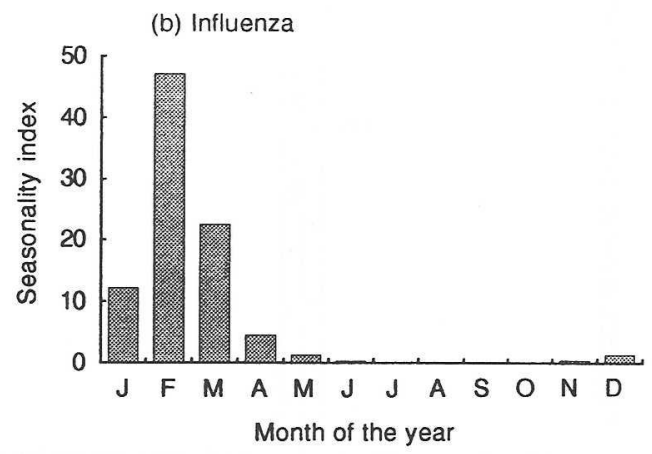

Fig. 3 Seasonality indices of months of the year

(a) Measles

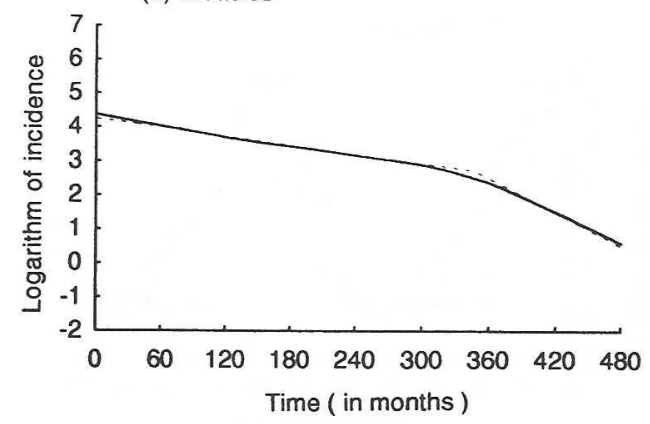

(b) Influenza

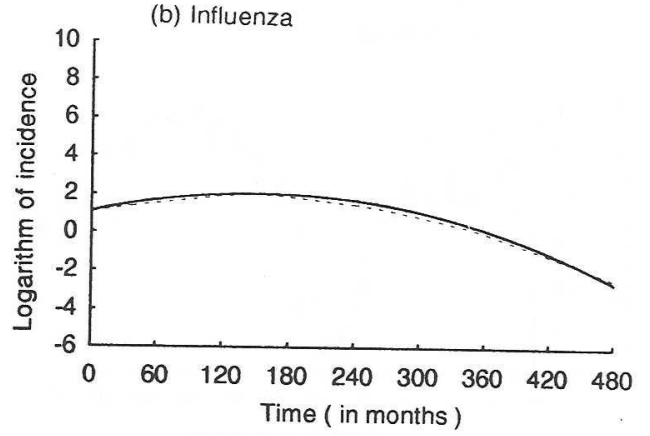

Fig. 4 Long-term incidence trends

The bold line was obtained by LOWESS and the thin, broken line was the corresponding trend line. 
(Fig. 5 (b)). Primary prediction values were compared with the observed data for 1989 and 1990 as shown in Fig. 6. A cyclic period of 36 months (3 years) has been observed in recent measles incidence after the introduction of vaccination for measles ${ }^{9}$. Though 1990 was the most prevalent year among every third year, the predicted values were always below the observed values. This indicates that there is a room for improvements in prediction. As to influenza, there was no such apparent tendency though performance of the prediction was not as suf-

(a) Measles

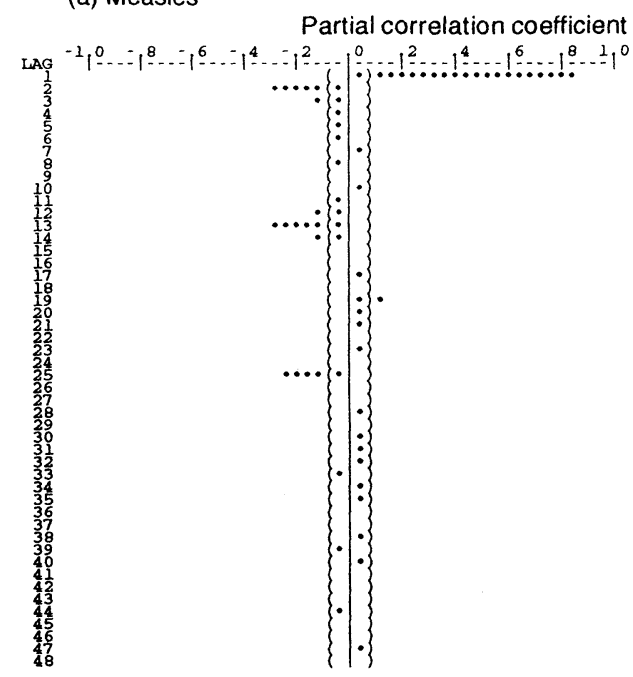

ficient as in the case of measles.

\section{Elaborated Prediction Models}

To improve the method, we constructed a model that involved previous residuals as explanatory variables for the present residual, i.e., autoregression. In these elaborated prediction models, lag 25 of measles was excluded because its effect turned out not to be significant. The $\mathrm{R}^{2} \mathrm{~S}$ were improved to 0.97 for measles and 0.79 for influenza. This improvement can also be visually recognized as illustrated in Fig. 6 .

(b) Influenza

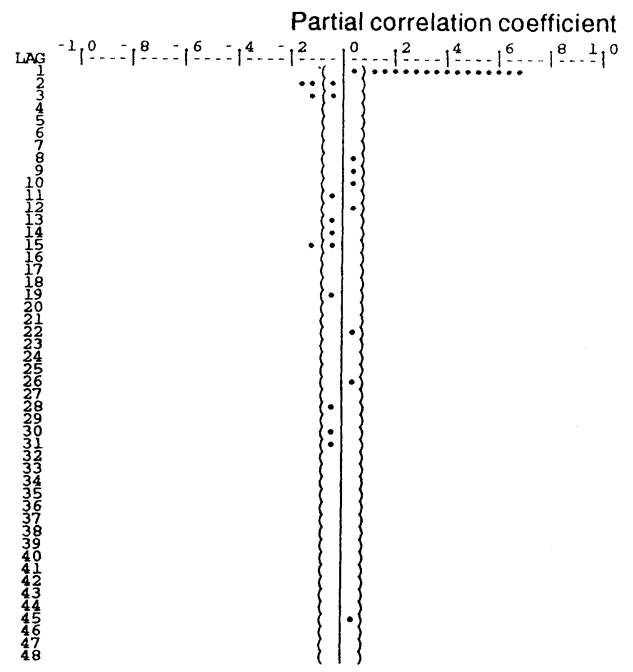

Fig. 5 PACF plot (partial autocorrelation function plot) of the residuals of the primary prediction

(a) Measles

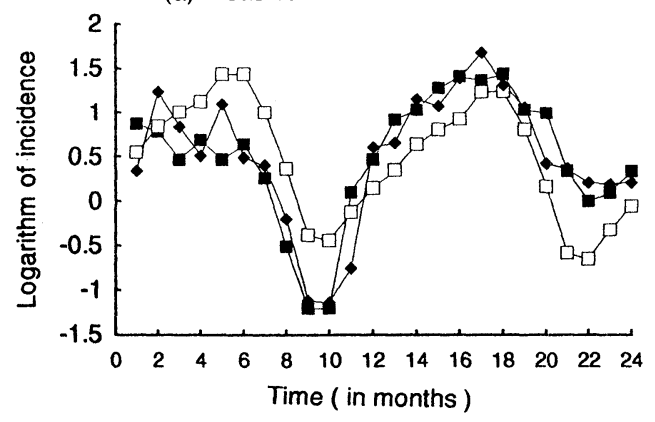

(b) Influenza

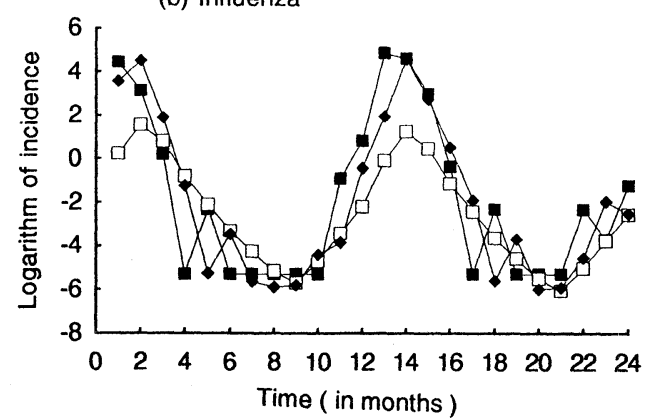

Fig. 6 Prediction on the incidences of 1989 and 1990

The observed incidence is denoted by solid squares. The open squares represent results of the primary prediction. The solid diamonds show the prediction by the elaborated method. 


\section{Discussion}

In the short-term prediction of infectious disease incidence, the statistical models used in this paper achieved a satisfactory outcome. The reason why the prediction for influenza was more difficult might be partly due to the existence of different virus types and temporal changes of these types. Relations to environmental conditions, e.g., temperature, humidity, etc., were recently studied in the prediction of infectious disease incidence ${ }^{10)}$. Although these factors on average were involved in our model as seasonality, variation of them year by year is important in predicting incidence. Since variations cause discrepancies in prediction, residuals of prediction might contain important information. Lags used in elaborated models might have improved predictive ability in this manner, though we cannot specify actual working factors.

It is of ten noted that the cases reported in the source data might be neither complete nor accurate enough. In spite of these inaccuracies, whether or not they existed, the methodology established here seemed to perform quite well.

Incidence is also affected by the change in age distribution of the Japanese population and the incidence data used in our analysis probably involved bias. According to our preliminary analysis using age-adjusted incidence data for measles and influenza from 1970 to 1990 (21 samples), the age-adjusted incidence and crude incidence were shown to be closely correlated, with correlation coefficients of more than 0.99. The effect of age-adjustment was small for influenza incidence. For measles incidence, the age-adjusted incidence could be well described with the crude incidence and a linearly increasing component with time (the square of the multiple correlation coefficient was 0.999 ). In general, it would be better to use age-adjusted rates in comparison between populations with different age distributions. Unfortunately, age-specific incidence data were only available annually, not monthly, in our source. Thus age-adjusted incidence data could not be used in our seasonality analysis. Despite the general recommedation for age-adjusted rates, use of crude rates here cannot be a serious defect because of the high correlation between age-adjusted and crude rates and incorporation of long-term trends in our models. This is because the effect of the change in age distribution can be suitably involved in the trend term as far as the effect in the interval can be approximately described by a line. Moreover, use of crude incidence is convenient and suitable from a practical viewpoint.

For infectious diseases other than measles and influenza, the same method can, in principle, be applied. We used here not the surveillance data but the data from Statistics on Communicable Diseases because it was advantageous to analyze long-term trends. Thus, some modification of the method might be required when we analyze the surveillance data. Such a possibility may well be true for diseases of which the numbers of reported cases are very small. A method more suitable to such cases has already been proposed and applied to disease-incidence data ${ }^{11}$. Recently, infectious disease incidence data were used to analyze whether or not fluctuations were caused by the deterministic relationship known as 'chaos' 12,13). According to the results obtained by the analysis, measles exhibited low-dimensional chaos on a city-by-city scale, whereas it exhibited a periodic cycle with noise on a larger, country -wide scale ${ }^{14}$ ). These aspects should be considered when we extend our analysis to the surveillance data.

In our analysis the observed trends were remained unanalyzed. In such an analysis, mathematical models are expected to play an important role rather than statistical models. Simple mathematical models have been used in ecology and their importance has been recognized. The three-year cycle observed for measles was well explained by such an approach ${ }^{9}$. Such cycles are of ten observed in ecological systems of host and 
parasite. These models can be applied to an analysis of long term trends involving evolutionary change ${ }^{15)}$. It was pointed out that epidemiology, which so far uses mainly statistical models, and ecology, which uses simple mathematical models, can develop together by communicating with each other ${ }^{16)}$. Moreover, the importance of such mathematical models in public health is going to be widely recognized ${ }^{17)}$.

To facilitate practical use of surveillance data, we finally propose the TS-decomposition diagram based on the analysis of trend (T) and seasonality $(\mathrm{S})$. In the diagram, observed and predicted incidence data are plotted on the plane where the trend and the seasonality are superimposed. Examples are illustrated in Fig. 7. The bold and almost plain line represents the longterm trend. The other three lines are a trend plus seasonal change line (i.e., prediction by the primary prediction model) with lines for $\pm 2 \mathrm{SD}$ shifts. Here SD represents the standard deviation of the residuals. The observed incidence in 1989 is represented by solid squares, predicted values by the elaborated models for the first half of 1990 by open squares. The prediction can be regarded as being made at the end of December, 1989, because data for 1990 were not used in the prediction. This was enabled by using values obtained in the elaborated prediction in place of the observed values.

(a) Measles

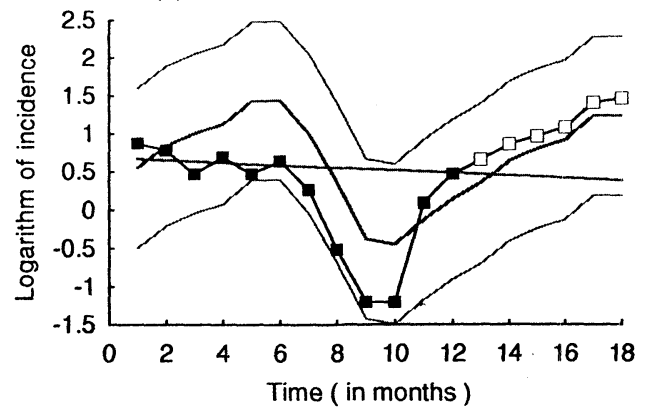

When we look at the TS-decomposition diagram of a certain infectious disease, we can immediately see whether or not it is more frequent in this year by comparing observed data with the trend plus seasonal change line. We can also see whether or not it is more frequent in a particular season of the year. Almost all the data should be located between the \pm 2 SD lines. Viewing data with this background information will help us to understand the behavior of the recent incidence data.

Statisical analysis of the incidence of infectious diseases will be useful when the committee for tuberculosis and infectious disease surveillance makes comments on the present situation of infectious disease incidence. Moreover, a report on infectious disease surveillance will be expected to draw more attention from a wider audience by using the TS-decomposition diagrams. This can be a useful tool in the health promotion of the community.

\section{Acknowledgments}

We are grateful to Prof. K. Ueda, Hiroshima University, for providing us valuable information on the infectious disease surveillance system in Hiroshima. We are also grateful to Prof. T. Yanagimoto for his valuable comments on the earlier version of this work. This work was partly supported by Grant-in-Aid for Scientific Research

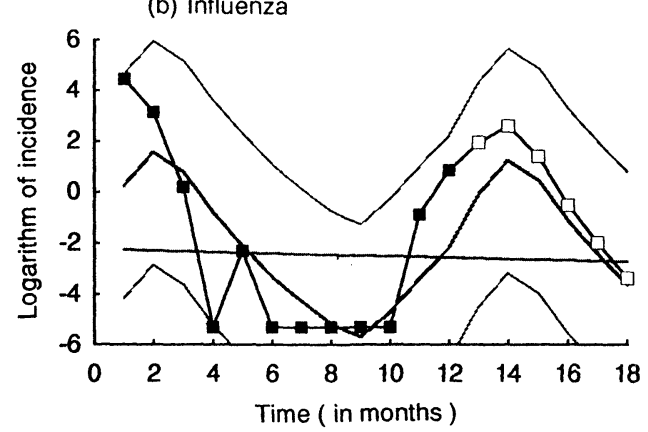

Fig. 7 TS-decomposition diagram

The observed incidence in 1989 represented by the solid squares is followed by a half-year prediction for 1990 represented by the open squares. Superimposed are the trend and the seasonality. See text for details . 
from the Ministry of Education, Science and Culture.

\section{References}

1) Tsuru, S., Seo, A., Kakehashi, M., Yoshinaga, F. and Tsubota, N.: Survey of attitudes among general practitioners regarding the introduction of a personal computer medical network system in community health care, Jpn. J. Public Health, 38, 472-482 (1991). (in Japanese with English summary)

2 ) Kakehashi, M., Tsuru, S., Seo, A. and Yoshinaga, F.: Present situation and future perspective on medical information network systems for community health using personal computers and IC cards. (Submitted, in Japanese with English summary)

3 ) Health and Welfare Statistics Division, Minister's Secretariat, Ministry of Health and Welfare: Statistics of Communicable Diseases and Food Poisoning Japan 1971, pp.25, 27, Health and Welfare Statistics Association, Tokyo (1973).

4 ) Statistics and Information Department, Minister's Secretariat, Ministry of Health and Welfare: Statistics on Communicable Diseases Japan 1989, pp.55, 57, Health and Welfare Statistics Association, Tokyo (1991).

5 ) Statistics and Information Department, Minister's Secretariat, Ministry of Health and Welfare: Statistics on Communicable Diseases Japan 1990, pp.55, 57, Health and Welfare Statistics Association, Tokyo (1991).

6 ) Wilkinson, L. : SYSTAT: The System for Statistics, Systat, Inc., Evanston (1989).

7 ) Kakehashi, M., Tsuru, S., Seo, A., Amran, A. and Yoshinaga, F. : Prediction on incidence of communicable diseases: Measles and influenza, 15-22, Proc. 2nd JapanKorea Biostat. Conf . (1992) .

8 ) Cleveland, W.S. : Robust locally weighted regression and smoothing scatterplots, J. Am. Stat. Assoc., 74, 829-836 (1979).

9 ) Kakehashi, M.: An analysis of the effect of measles vaccination program by a mathematical model, Jpn. J. Public Health, 37, 481 -490 (1990). (in Japanese with English summary)

10) Shoji, M., Tsunoda, A. and Ishida, N.: Correlation between the occurrence of infantile infectious diseases and the weather, Kosankinbyo Kenkyusho Zasshi, 38, 91-101 (1986) . (in Japanese with English summary)

11) Kashiwagi, N. and Yanagimoto, T.: Smoothing serial counts data through a state-space model, Biometrics, (to appear).

12) Olsen, L.F., Truty, G.L. and Schaffer, W.M.: Oscillations and chaos in epidemics: A nonlinear dynamic study of six childhood diseases in Copenhagen, Denmark, Theor. Popul. Biol. , 33, 344-370 (1988).

13) Sugihara, G. and May, R.M.: Nonlinear forecasting as a way of distinguishing chaos from measurement error in time series, Nature, 344, 734-741 (1990) .

14) Sugihara, G., Grenfell, B. and May, R.M. : Distinguishing error from chaos in ecological time series, Philos. Trans. R. Soc. Lond. B, 330, 235-251 (1990).

15) Kakehashi, M. and Yoshinaga, F.: Evolution of airborne infectious diseases according to changes in characteristics of host population, Ecol. Res. , 7, 235-243 (1992).

16) Anderson, R.M.: Population and infectious diseases: Ecology or epidemiology?, J. Anim. Ecol. , 60, 1-50 (1991).

17) Anderson, R.M. and Nokes, D.J. : Mathematical models of transmission and control, In "Oxford Textbook of Public Health 2nd ed. Vol. 2" (Editors: Holland, W.W., Detels, R. and Knox, G.), pp.225-252, Oxford Univ. Press, Oxford (1991).

(Received May 6, 1992/Accepted November 24, 1992) 